\section{Taxotere ${ }^{\circledR}$ (Docetaxel) verbessert signifikant das Überleben von Patienten mit fortgeschrittenem Prostatakarzinom}

Auf dem diesjährigen Symposium der ASCO zum Prostatakarzinom gab das Unternehmen sanofi-aventis Ergebnisse zum Langzeitüberleben aus der großen Phase-III-Studie TAX 327 bekannt. In der TAX 327 erhielten Patienten mit metastasiertem hormonrefraktären Prostatakarzinom (mHRPC) entweder alle drei Wochen $75 \mathrm{mg} / \mathrm{m}^{2}$ Taxotere ${ }^{\circledR}$ (Docetaxel) in Kombination mit Prednison oder ein Mitoxantron-Prednison-Schema. Dabei zeigt sich ein Gesamtüberlebensvorteil im Taxotere-Arm mit einer medianen Überlebenszeit von 19,3 Monaten gegenüber 16,3 Monaten, $p=0,005$. Dies entspricht einer Reduktion des Mortalitätsrisikos um 21\% für die Patienten, die dreiwöchentlich mit $75 \mathrm{mg} / \mathrm{m}^{2}$ Taxotere und Prednison behandelt wurden. Diese Ergebnisse bestätigen die Daten der Erstanalyse.

Der Überlebensvorteil war unabhängig von folgenden Merkmalen: Alter, vorhandener oder nicht vorhandener Schmerzsymptomatik, dem PSA-Ausgangswert, sowie den Ausgangswerten für Performance und Lebensqualität, gemessen mit dem FACT-P.

Die Ergebnisse waren hochsignifikant bei Patienten mit einem hohen PSA-Ausgangswert ( $\geq 115 \mathrm{ng} / \mathrm{ml}: 17,5$ Monate gegenüber 12,8 Monaten, $p=0,008$ ), bei Patienten ohne Schmerzen (23,0 Monate gegenüber 19,8 Monaten, $p=0,009$ ), bei Patienten mit einem guten Performancestatus ( $\geq 90 \%$ : 22,8 Monate gegenüber 19,8 Monaten, $\mathrm{p}=0,011$ ) und bei Patienten mit schlechterer Lebensqualität (FACT-P < 109: 18,3 Monate gegenüber $12,4$ Monaten, $p=0,002)$. Statistische Signifikanz hinsichtlich des medianen Überlebens wurde nicht erreicht bei höherem Alter, einem PSA-Wert $<115 \mathrm{ng} / \mathrm{ml}$, vorhandenen Schmerzen, einem Performancestatus $\leq 80 \%$ und einem Wert für Lebensqualität $\geq 109$ im FACT-P.

Weitere Informationen bei Sanofi-Aventis Deutschland GmbH Brand \& Scientific Communication Cristina Iannazzo

Industriepark Höchst

Gebäude F821

65926 Frankfurt am Main

Tel. +49 69 305-24417, Fax -18802

www.sanofi-aventis.de

\section{EAU empfiehlt Sunitinib als Erstli- nien-Therapie bei fortgeschrittenem und/oder metastasiertem Nierenzellkarzinom}

In ihren neuen Leitlinien hat die EAU (European Association of Urology) Sunitinib als Erstlinien-Therapie bei fortgeschrittenem und/ oder metastasiertem Nierenzellkarzinom (mRCC) für Patienten mit niedrigem und mittlerem Risiko empfohlen. Damit rückt Sunitinib in die vorderste Reihe bei der medikamentösen Therapie dieser schwer zu behandelnden Krankheit. Die EAU-Leitlinien resultieren aus einer umfangreichen, unabhängigen Bewertung klinischer Studiendaten und weiterer Quellen, die in Zusammenhang mit der Behandlung des mRCC stehen. Sie sollen
Urologen ein Evidenz-basiertes Management bei der Behandlung des Nierenzellkarzinoms erleichtern.

Die uneingeschränkte Zulassung von Sunitinib zur Erstlinien-Therapie des mRCC basiert auf den Ergebnissen einer internationalen Phase-III-Studie. In diese Multicenter-Studie waren 750 Patienten eingeschlossen, die randomisiert entweder Sunitinib oder IFN- $\alpha$ erhielten. Patienten, die Sunitinib erhielten, lebten im Median elf Monate progressionsfrei. Das ist mehr als doppelt so lang wie die fünf Monate, die unter IFN- $\alpha$ erreicht wurden.

Als Multi-Tyrosinkinase-Inhibitor greift Sunitinib gezielt in verschiedene Signalwege des Tumors ein; dadurch wird nicht nur die Angiogenese, sondern auch die Proliferation, also die Vermehrung der Krebszellen selbst ge- hemmt. Das Medikament wird oral verabreicht, was die Behandlung für Patient und Arzt gleichermaßen erleichtert.

Die unter Sunitinib auftretenden unerwünschten Nebenwirkungen sind in der Regel beherrschbar. Unabhängig vom Schweregrad wurden am häufigsten (bei mindestens 20\% der Patienten) u. a. Erschöpfung, Magen-Darm-Beschwerden wie Durchfall, Übelkeit, Schleimhautentzündungen, Oberbauchbeschwerden und Erbrechen, Hautverfärbungen, Geschmacksstörungen und Appetitlosigkeit beobachtet.

Weitere Informationen bei

Pfizer Deutschland GmbH

Franziska Theobald

Tel. +49 72161 01-9466

franziska.theobald@pfizer.com

\title{
PharmaTicker+++ PharmaTicker+++ PharmaTicker+++ PharmaTicker+++
}

GlaxoSmithKline GmbH \& Co. KG. Das Unternehmen hat den Start einer Phase-III-Studie zur Überprüfung der Wirksamkeit von Lapatinib bei Kopf-Hals-Tumoren bekannt gegeben. In diese klinische Studie werden weltweit 680 Hochrisiko-Patienten mit lokal fortgeschrittenem Kopf-Hals-Tumor eingeschlossen (Stadien II, III und IV), die bereits voroperiert sind.

Weitere Informationen bei

GlaxoSmithKline GmbH \& Co. KG

Arnd Prilipp

Tel. +49 8936044 - 8117

arnd.prilipp@gsk.com
Merck Pharma GmbH. Das neue Hörbuch «Leben mit Darmkrebs - zuhören, begreifen, handeln» wurde von Merck in Zusammenarbeit mit verschiedenen Spezialisten speziell für Darmkrebspatienten und deren Angehörige entwickelt. Das Hörbuch ist eine ganz neue und moderne Form der Patienteninformation und erläutert die heute üblichen Behandlungsmethoden.

Kostenlose Anforderung unter

Tel. +49 6151 72-0 (montags - freitags von 8 - $18 \mathrm{Uhr}$ ) service@merck.de

(mit Angabe der gewünschten Anzahl)
Cephalon Pharma GmbH. In der Primärtherapie der akuten promyelozytären Leukämie (APL) steigert Arsentrioxid (Trisenox ${ }^{\circledR}$ ) das krankheitsfreie sowie das Gesamtüberleben im Vergleich zur alleinigen Standardchemotherapie. Arsentrioxid ist seit 2002 von der EMEA für die Salvagetherapie der APL zugelassen und wird erfolgreich zur Induktion einer Remission und Konsolidierung bei erwachsenen Patienten mit rezidivierender oder refraktärer APL eingesetzt.

Weitere Informationen be

Cephalon Pharma Gmbh fsalomon@cephalon.com 


\section{Deutscher Schmerztag: Experten plädieren für flexible Dosierung und Applikation}

Die Arzneimittelkommission der deutschen Ärzteschaft (AkdÄ) empfiehlt in ihren neuen Leitlinien zur Tumorschmerz-Therapie eine individuell auf den Patienten abgestimmte Analgesie. «Das ist wichtig, weil circadiane Rhythmen zu Variationen im Schmerzempfinden führen», sagt Dr. Uwe Junker vom Sana-Klinikum in Remscheid im Rahmen des diesjährigen Deutschen Schmerztages (15. bis 17. März 2007) in Frankfurt. Solche Schwankungen lassen sich laut Experte ideal mit der zweimal täglichen Gabe eines oralen, retardierten Opioids, gegebenenfalls in unterschiedlicher Wirkstärke, lindern. Das bestätigt eine Multicenterstudie von Dr. Till Wagner: Bei 658 der 1.286 Probanden variierte die Schmerzstärke am Tag und in der Nacht. Tumorpatienten litten meist nachts an stärkeren Schmerzen und erhielten somit abends eine höhere Palladon ${ }^{\circledR}$ _ Dosis. Multimorbide Patienten hingegen empfanden ihre Schmerzen tagsüber als stärker und bekamen morgens eine stärkere Analgesie. Nach sieben Tagen hatten sich bei über 92 Prozent aller Patienten nicht nur die tageszeitlichen Schwankungen im Schmerzempfinden reduziert, auch die Schmerzstärke war geringer. Die Lebensqualität verbesserte sich um mehr als die Hälfte, nachdem sie morgens und abends
Palladon ${ }^{\circledR}$ retard in asymmetrischer, schmerzangepasster Dosierung erhalten haben.

Bedarfsadaptierte Invasivtherapie erhöht Lebensqualität

«Ist eine flexible orale Schmerzmedikation nicht möglich, sollte die parenterale Applikation gewählt werden», sagt Dr. HansBernd Sittig vom Medizinischen Versorgungszentrum Buntenskamp in Geesthacht. Zum Beispiel wenn Tabletten, Kapseln und Tropfen nicht toleriert werden oder bei Palliativpatienten in der Terminalphase. Hat der Patient Schluckstörungen, treten Nebenwirkungen wie Nausea und Emesis auf oder besteht ein sehr hoher AnalgetikaBedarf, müssen Schmerzmittel ebenfalls parenteral gegeben werden.

\section{Palladon ${ }^{\circledR}$ injekt in drei Dosierungen}

Doch bislang war eine HydromorphonInjektion nur in der $2 \mathrm{mg} / 1 \mathrm{ml}$-Dosierung erhältlich und der Einsatz in der Dauertherapie somit begrenzt. Seit April 2007 gibt es von Mundipharma das neue Palladon ${ }^{\circledR}$ injekt. Dieses parenterale Hydromorphon für Patienten mit starken chronischen oder Tumorschmerzen ist in drei Dosierungen vorhanden: Die $2 \mathrm{mg} / 1 \mathrm{ml}-$ Ampulle eignet sich als Einmalgabe oder Einstiegsdosis, um starke Schmerzen über einen kurzen Zeitraum schnell zu lindern. So zum Beispiel posttraumatisch, bei postoperativen Schmerzen (erst 24 Stunden nach der Operation) oder zur Titration und Dosiseinstellung. Die
10mg/1ml-Ampulle reicht meist als Tagesdosis aus. Die $100 \mathrm{mg} / 10 \mathrm{ml}-$ Dosierung ist eine Wochendosis oder für die Dauerbehandlung mit Pumpen- und Portsystemen. Da für Port- und Pumpen-Füllungen mit Hydromorphon nur ein geringeres Applikationsvolumen erforderlich ist, ist Palladon ${ }^{\circledR}$ injekt optimal für Patienten, bei denen das subkutane Bolusoder Infusionsvolumen minimal sein muss. Auch das aufwändige Brechen zahlreicher Ampullen zum Befüllen von Pumpen- und Ports entfällt.

Die Wirkung tritt bei intravenöser Gabe bereits nach fünf beziehungsweise bei subkutaner Gabe nach fünf bis zehn Minuten ein und hält drei bis vier Stunden an. Hydromophon bildet keine aktiven Metabolite wie Morphin-6-glucuronid und wird unabhängig vom Cytochrom-P450-System der Leber metabolisiert. Damit ist es auch bei Niereninsuffizienz und Multimedikation geeignet. Die Injektions-Lösung hat einen $\mathrm{pH}$-Wert von 4,0 und ist lokal gut verträglich. «Palladon ${ }^{\circledR}$ injekt schließt eine therapeutische Lücke», sagt Sittig, «denn viele Patienten können nun weiterhin von Hydromorphon profitieren, auch wenn eine orale Therapie mit retardiertem Palladon ${ }^{\circledR}$ nicht mehr möglich ist».

Weitere Informationen bei

Mundipharma

Vertriebsgesellschaft mbH \& Co. KG Gebührenfreie Infoline 0800855111 info@mundipharma.de www.mundipharma.de

\section{PharmaTicker+++ PharmaTicker+++ PharmaTicker+++ PharmaTicker+++}

Baxter Deutschland GmbH. Soeben wurde das neue Programmheft des Baxter Competence Centers veröffentlicht. Auch im Jahr 2007 steht eine große Vielfalt an Fortbildungen aus unterschiedlichsten Fachgebieten für Ärzte, Apotheker und Pflegekräfte zur Verfügung. Das komplette Programmheft kann bestellt werden unter baxter@the-messengers.de.

Anmeldung zu den Veranstaltungen unter

Tel. +496172 2851014

baxter@the-messengers.de

www.baxter.de
B. Braun Melsungen AG. Mit Softa-Man acute bietet die B. Braun Melsungen AG jetzt aktuell ein Händedesinfektionsmittel mit einer umfassenden viruziden Wirksamkeit von einer Minute an. Es ist damit sehr schnell wirksam, sicher und anwenderfreundlich. Der Wirkstoffgehalt von Softa-Man acute ist mit $63 \%$ wesentlich niedriger als der von herkömmlichen Präparaten. Außerdem ist es parfüm- und farbstofffrei.

Weitere Informationen bei

B. Braun Melsungen AG

Hotline 0800-2272868

kundenservice@bbraun.com
Sanofi Pasteur MSD GmbH. Die Ständige Impfkommission (STIKO) am Robert Koch-Institut in Berlin hat die Impfung gegen humane Papillomviren (HPV) als Standardimpfung empfohlen und damit in den Impfkatalog aufgenommen. Gardasi ${ }^{\circledR}$ von Sanofi Pasteur MSD ist bislang der einzige in Deutschland zugelassene Impfstoff zur Prävention von Gebärmutterhalskrebs und anderen HPV-assoziierten Erkrankungen.

Weitere Informationen bei

Sanofi Pasteur MSD GmbH

Michael Kölsch

Tel. +49 6224 594-233, Fax -161

MKoelsch@spmsd.com 